attempt to use the whole population. If, in addition, there are people willing to participate, then one has a "living laboratory'. With a female population of 20,000 over 20 years of age yielding four or five epidermoid cancers of the cervix uteri annually, it is possible to screen by the cervical smear technique in a few years the 17,000 women between 20 and 70 years of age, and note the effect on the annual incidence of cervical carcinomas. Once the facilities for taking and examining the smears are provided it is necessary to persuade the women to come. The cancer educationalists' propaganda, using the mass media and lectures to women's organizations, forms a useful background. Only by working through their general practitioners is it possible, however, to persuade the bulk of the women to attend for the taking of the smears. In order to get the general practitioners' cooperation, it is necessary to give evidence of the value of the screening.

\section{Some Aspects of Medical Genetics in Island} Populations

\section{A. CLARKE (Liverpool)}

The study of island populations is of considerable potential interest for the investigation of genetic problems. There may be an increase in consanguineous marriages but popular guesses as to the extent to which breeding groups on an island are closed are often wrong, and this can be checked against parish register material. Furthermore, as was found in Japan, inbreeding may not necessarily be deleterious. The pattern of congenital malformations may be different from that of the mainland and a register of these should be kept, as among other things this is a useful stimulator not only of further research but also of better social care. Again, because of genetic drift, whereby chance exercises a considerable influence on gene frequencies, blood group distributions may be unusual and unfavourable mutations persist in isolated communities. These factors will be discussed in relation to the Isle of Man.

\section{Genetics Survey of the Manx}

R. J. MITCHELL. (Department of Anthropology, University of Durham)

In recent years physical anthropologists and human biologists have carried out numerous surveys of the genetical characteristics of populations. In such a context this paper is a preliminary report on a study of genetic variability in the Manx population. Included in the survey were those individuals having three or four grandparents born on the Isle of Man. Results are given for the following unifactorial traits: most of the blood group antigenic systems, including ABO, Rhesus, Kell, Duffy, $\mathrm{Lu}^{\mathrm{a}}$, and $\mathrm{Kp}^{\mathrm{a}}$, the red blood cell isoenzyme acid phosphatase, and the plasma proteins haptoglobin and transferrin. Non-serological inherited factors such as phenylthiocarbamide testing are also reported upon.

For some of the serological factors comparisons were made between blood donor and non-blood donor samples. Moreover the incidence of the genetic features was compared with those found in contiguous areas of Britain, particularly south west Scotland and Cumberland.

Mention is made of previous anthropological studies of the Island's population and their main findings. All previous work was of an anthropometric rather than serological nature. One aim of the present work is to discover whether genetic factors complement the variability in the Manx population found in the previous studies.

A very brief account of the main periods occurring in the Island's history is given because of its possible use in helping to interpret the results of the present survey.

\section{Erythroblastosis Foetalis}

A. E. ClaIREAUX (Department of Morbid Anatomy, Hospital for Sick Children, London)

Erythroblastosis foetalis (haemolytic disease of the newborn) is an important, if decreasing cause, of perinatal death. Results obtained during the perinatal mortality survey (1958) (Claireaux, 1963) showed that it was responsible for $4.0 \%$ of all perinatal deaths and the incidence was 1.4 per 1,000 live and stillbirths. The final results of a similar study, British births (1970), are not yet available but preliminary evaluation of postmortem material shows that it is still a factor in perinatal death but the incidence is probably lower than in 1958.

Erythroblastosis foetalis was a term coined in 1933 to include the three main types of the disease: $(a)$ hydrops foetalis; (b) icterus gravis neonatorum; (c) congenital haemolytic anaemia. This last is not to be confused with congenital spherocytosis. Hydrops foetalis was the most severe form of the disease and usually resulted in a stillbirth or in the delivery of a moribund infant. Icterus gravis was, io untreated, also likely to have a fata termination and the infant succumbed asc a result of brain damage (kernicterus). Theo remaining cases, much less severe and seldom fatal, were clinically regarded as $\frac{\bar{\omega}}{\overline{2}}$ examples of haemolytic anaemia. The $\overrightarrow{\widetilde{\sigma}}$ clinical features of the disease and the 0 pathological changes in fatal cases were known some considerable time before the discovery of the Rhesus blood groups. (1940). It was only then that it became $\overrightarrow{-}$ clear the condition resulted from iso- $\omega$ immunization of the mother by a blood group she did not possess.

\section{Reference}

Claireaux A. E (1963) In Modern Trends in Obstetrics, ed. R. J. Kellar, p. 191. Butter-6 worths, London.

The Role of RH Antibodies in Causing Haemolytic Disease of the Newborn and if
Preventing It

P. L. MOLLISON (Department of Haemato $\overrightarrow{0}$ tology, St Mary's Hospital Medical School, London)

HAEMOLYTIC DISEASE OF THE NEWBORN

The relation between the amount of anti $\mathrm{Rh}$ on the red cells of an Rh-positive infant and the severity of the haemolytic process is not very close; one reason mays be the varying ability of different examples of anti-Rh to bring about red cell destruction.

In haemolytic disease of the newborn the concentration of anti-R $h$ in the plasma of the Rh-positive infant is always very? much lower than that in the mother's plasma, emphasizing that the relativelyo slow transfer of IgG across the placenta 3 plays a crucial role in mitigating theo severity of the damage. Because of this slow transfer, it is quite safe to give in-o jections of the order of $200 \mu \mathrm{g}$ anti-Rh to Rh-negative women who are pregnant $N$ with an Rh-positive foetus. Incidentally, $N$ even if such a dose is accidentally in- $N$ jected into a newborn Rh-positive infant, $\omega$ it causes only very mild haemolytic disease.

PRIMARY RH IMMUNIZATION

A single injection of $R$ h-positive red cells induces primary immunization in abou? $65 \%$ of Rh-negative subjects. With a relatively large dose ( $200 \mathrm{ml}$ cells), anti-R $\overrightarrow{P B}$ is as a rule readily detectable three to si 